\title{
Asymptotic and resampling strategies for assessing and comparing indirect effects in multiple mediator models
}

\author{
Kristopher J. Preacher \\ University of Kansas, Lawrence, Kansas \\ AND \\ Andrew F. HaYes \\ Ohio State University, Columbus, Ohio
}

\begin{abstract}
Hypotheses involving mediation are common in the behavioral sciences. Mediation exists when a predictor affects a dependent variable indirectly through at least one intervening variable, or mediator. Methods to assess mediation involving multiple simultaneous mediators have received little attention in the methodological literature despite a clear need. We provide an overview of simple and multiple mediation and explore three approaches that can be used to investigate indirect processes, as well as methods for contrasting two or more mediators within a single model. We present an illustrative example, assessing and contrasting potential mediators of the relationship between the helpfulness of socialization agents and job satisfaction. We also provide SAS and SPSS macros, as well as Mplus and LISREL syntax, to facilitate the use of these methods in applications.
\end{abstract}

The behavioral science literature is replete with studies demonstrating that a particular independent variable explains variability in a dependent variable. Establishing relationships between variables is important, because correlation is a necessary (but not sufficient) condition for claiming that two variables are causally related. Of even greater scientific interest is explaining how or by what means a causal effect occurs. Questions about causeeffect relations invoke the idea of mediation, the process by which some variables exert influences on others through intervening or mediator variables. For example, evidence suggests that job autonomy, cognitive ability, and job-related skills all predict job performance. But it is even more informative to be able to claim that they exert their effects on job performance through role breadth - the variety of tasks a person performs on the job (Morgeson, Delaney-Klinger, \& Hemingway, 2005). Assad, Donnellan, and Conger (2007) found that the effect of optimism on romantic relationship quality is mediated by cooperative problem solving. Kalyanaraman and Sundar (2006) showed that perceived interactivity of a Web portal functions as a mediator of the effect of customization on attitudes toward the portal. Such hypotheses go beyond mere description and help to explain process and causality.

There exists a large and growing literature on methods of testing simple mediation hypotheses - those in which the effect of some causal variable $X$ on some proposed outcome $Y$ is mediated by a single variable $M$. Our focus in this article is to discuss and illustrate the application of some of these methods to the estimation and testing of mediated effects in multiple mediator models - those with more than a single proposed mediator variable. We then discuss how statistical contrasts of two or more indirect effects in a multiple mediator model may be conducted, and present SAS, SPSS, Mplus, and LISREL syntax to facilitate the testing of multiple mediation hypotheses.

\section{MEDIATION IN BEHAVIORAL RESEARCH}

Mediation hypotheses posit how, or by what means, an independent variable $(X)$ affects a dependent variable $(Y)$ through one or more potential intervening variables, or mediators $(M)$. We address only the situation in which the causal order of $X, M$, and $Y$ can be established on theoretical or procedural grounds. If a logical ordering of $X, M$, and $Y$ cannot be established, other methods should be used to investigate mediation (e.g., Azen, 2003).

Mediation processes involving only one mediating variable we term simple mediation. Figure 1B depicts a simple mediation model and shows how variable $X$ 's causal effect can be apportioned into its indirect effect on $Y$ through $M$ and its direct effect on $Y$ (path $c^{\prime}$ ). Path $a$ represents the effect of $X$ on the proposed mediator, whereas path $b$ is the effect of $M$ on $Y$ partialling out the effect of $X$. All of these paths would typically be quantified with unstandardized regression coefficients. The indirect effect of $X$ on $Y$ through 
A
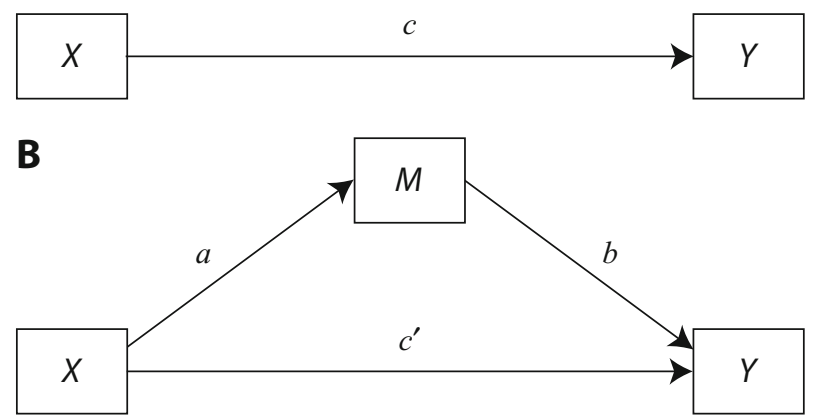

Figure 1. (A) Illustration of a direct effect. $X$ affects $Y$. (B) Illustration of a mediation design. $X$ is hypothesized to exert an indirect effect on $Y$ through $M$.

$M$ can then be quantified as the product of $a$ and $b$ (i.e., $a b$ ). The total effect of $X$ on $Y$ is quantified with the unstandardized regression weight $c$ (Figure 1A). The total effect of $X$ on $Y$ can be expressed as the sum of the direct and indirect effects: $c=c^{\prime}+a b$. Equivalently, $c^{\prime}$ is the difference between the total effect of $X$ on $Y$ and the indirect effect of $X$ on $Y$ through $M$ - that is, $c^{\prime}=c-a b$. These identities hold in regression and structural equation modeling (SEM) where $M$ and $Y$ are continuous, but not in cases where one or more of the dependent variables are binary; such situations require logistic or probit regression, in which case the identity does not hold (MacKinnon \& Dwyer, 1993).

At least a dozen methods for testing hypotheses about mediation have been proposed (see MacKinnon, Lockwood, Hoffman, West, \& Sheets, 2002, for an overview). By far the most commonly used is the causal steps strategy, popularized by Baron and Kenny (1986), in which the investigator estimates the paths of the model in Figure 1, using OLS regression or SEM, and assesses the extent to which several criteria are met. Variable $M$ is a mediator if $X$ significantly accounts for variability in $M, X$ significantly accounts for variability in $Y, M$ significantly accounts for variability in $Y$ when controlling for $X$, and the effect of $X$ on $Y$ decreases substantially when $M$ is entered simultaneously with $X$ as a predictor of $Y$. As Kenny, Kashy, and Bolger (1998) note, however, the latter criterion will be satisfied when the first and third criteria are satisfied and when the signs of the effects are consistent with the proposed mediation process. With reference to Figure 1, these criteria essentially require paths $a, b$, and $c$ to be significant and $c^{\prime}$ to be smaller than $c$ by a nontrivial amount. However, some authors (Collins, Graham, \& Flaherty, 1998; Judd \& Kenny, 1981; Kenny et al., 1998; MacKinnon, 1994, 2000; MacKinnon, Krull, \& Lockwood, 2000; Shrout \& Bolger, 2002) have argued that a significant total effect of $X$ on $Y$ (quantified as $c$ in Figure 1) is not necessary for mediation to occur.

Most other approaches to testing mediation hypotheses focus not on the individual paths in the mediation model but instead on the product term $a b$, under the logic that this product is equal to the difference between the total and direct effect. The Sobel test (Sobel, 1982, 1986), also called the product-of-coefficients approach, involves computing the ratio of $a b$ to its estimated standard error $(S E)$. Numerous formulas have been proposed for estimating this $S E$ (MacKinnon et al., 2002; Preacher \& Hayes, 2004), but the differences among them usually have negligible effects on test outcomes. A $p$ value for this ratio is computed in reference to the standard normal distribution, and significance supports the hypothesis of mediation.

Methodologists have taken issue with the use of the standard normal distribution for deriving a $p$ value for the indirect effect, since the sampling distribution of $a b$ is normal only in large samples. One strategy, the distribution of the product approach (see MacKinnon et al., 2002; MacKinnon, Lockwood, \& Williams, 2004), bases inference on a mathematical derivation of the distribution of the product of two normally distributed variables (Aroian, 1947; Craig, 1936; MacKinnon et al., 2004; Springer, 1979) and thus acknowledges the skew of the distribution of products rather than imposing the assumption of normality. SPSS, SAS, and $\mathrm{R}$ code are available for generating confidence intervals (MacKinnon, Fritz, Williams, \& Lockwood, 2007).

Bootstrapping, a nonparametric resampling procedure, is an additional method advocated for testing mediation that does not impose the assumption of normality of the sampling distribution. Bootstrapping is a computationally intensive method that involves repeatedly sampling from the data set and estimating the indirect effect in each resampled data set. By repeating this process thousands of times, an empirical approximation of the sampling distribution of $a b$ is built and used to construct confidence intervals for the indirect effect. Because we advocate bootstrapping for testing indirect effects in multiple mediator models later in this article, we will save a discussion of the technicalities of the method until then. For details of its application to simple mediation models, see Bollen and Stine (1990), Lockwood and MacKinnon (1998), MacKinnon et al. (2004), Shrout and Bolger (2002), and Preacher and Hayes $(2004,2008)$.

In extensive sets of simulations, MacKinnon et al. (2002; MacKinnon et al., 2004) examined the performance of these methods (among others) to assess their Type I error rates and power. They recommended the use of the distribution of the product approach or bootstrapping over the Sobel test or causal steps approach, on the grounds that the former have higher power while maintaining reasonable control over the Type I error rate. Even though it is the most commonly used method, the causal steps strategy cannot be recommended except in large samples. For a discussion, see Preacher and Hayes (2004) and MacKinnon et al. (2002).

\section{STRATEGIES FOR ASSESSING INDIRECT EFFECTS IN MULTIPLE MEDIATOR MODELS}

A design that has received less attention in both the methodological and applied literature involves simultaneous mediation by multiple variables, or multiple mediation. Researchers often have several putative mediators in mind to account for a given $X \rightarrow Y$ relationship. For example, Aiken, West, Woodward, Reno, and Reynolds (1994) examined the mediating effects of four perceptions 
(perceived susceptibility to breast cancer, perceived severity of consequences of breast cancer, perceived benefits of mammography, and barriers to obtaining a mammogram) on the effectiveness of educational programs designed to increase mammography screenings. Reynolds et al. (2004) explored knowledge, availability of fruits and vegetables, and parental consumption as mediators of the effect of a school-based intervention to increase healthy food consumption on children's consumption of fruits and vegetables. Raver and Gelfand (2005) hypothesized that the relationship between ambient sexual harassment and team financial performance is mediated by team conflict, team cohesion, and team citizenship. Constantine (2007) examined the mediating roles of general and multicultural counseling competence and client-counselor therapeutic alliance in the relationship between subtle racist denigration and satisfaction with counseling. Carson, Carson, Gil, and Baucom (2007) showed that the effectiveness of a mindfulness-based relationship enhancement intervention on relationship satisfaction was mediated by partners' perceptions of engaging in exciting self-expanding activities, but not by partners' tolerance of each other's difficult characteristics, or the ability to relax. Holbert, Shah, and Kwak (2003) illustrated that viewing traditional dramas, progressive dramas, and situation comedies mediated the influence of political ideology on support for women's rights. Also, de Zavala and Van Bergh (2007) demonstrated that the effect of the need for closure on political conservatism was mediated by traditional and modern worldviews, but not by a postmodern worldview. Other examples are readily found.

We suspect that the main reason there has been little focus on methods for testing multiple mediation hypotheses is that the analytic methods are somewhat arcane, relative to those for simple mediation. To date, only a few authors (e.g., Bollen, 1987, 1989; Brown, 1997; Cheung, 2007; MacKinnon, 2000; West \& Aiken, 1997) have devoted attention to the simultaneous testing of multiple indirect effects, yet the potential uses for such methods are clear and abundant.

Figure 2 depicts a multiple mediation model with $j$ mediators. ${ }^{1}$ As in Figure 1, Figure 2A represents the total effect of $X$ on $Y$ (path $c$ ). Figure 2B represents both the direct effect of $X$ on $Y$ (path $c^{\prime}$ ) and the indirect effects of $X$ on $Y$ via the $j$ mediators. The specific indirect effect of $X$ on $Y$ via mediator $i$ (Brown, 1997; Fox, 1985) is defined as the product of the two unstandardized paths linking $X$ to $Y$ via that mediator. For example, the specific indirect effect of $X$ on $Y$ through $M_{1}$ is quantified as $a_{1} b_{1}$. The total indirect effect of $X$ on $Y$ is the sum of the specific indirect effects, $\Sigma_{i}\left(a_{i} b_{i}\right), i=1$ to $j$, and the total effect of $X$ on $Y$ is the sum of the direct effect and all $j$ of the specific indirect effects: $c=c^{\prime}+\Sigma_{i}\left(a_{i} b_{i}\right), i=1$ to $j$. The total indirect effect can also be calculated as $c-c^{\prime}$.

There are several advantages to specifying and testing a single multiple mediation model in lieu of separate simple mediation models. First, testing the total indirect effect of $X$ on $Y$ is analogous to conducting a regression analysis with several predictors, with the aim of determining whether an overall effect exists. If an effect (in this case mediation) is found, one can conclude that the set of $j$ variables mediates the effect of $X$ on $Y$. Second, it is possible to determine to what extent specific $M$ variables mediate the $X \rightarrow Y$ effect, conditional on the presence of other mediators in the model. Third, when multiple putative mediators are entertained in a multiple mediation model, the likelihood of parameter bias due to omitted variables is reduced. By contrast, when several simple mediation hypotheses are each tested with a simple mediator model, these separate models may suffer from the omitted variable problem, which can lead to biased parameter estimates (Judd \& Kenny, 1981). Fourth, including several mediators in one model allows the researcher to determine the relative magnitudes of the specific indirect effects associated with all mediators. In other words, including several mediators in the same model is one way to pit competing theories against one another within a single model. Theory comparison is good scientific practice.

Investigating multiple mediation in the context of models like those depicted in Figure 2 can be considerably more complex than investigating simple mediation. Assessing multiple mediation involves not only deciding whether or not an indirect effect exists, but also deciding how to tease apart individual mediating effects often attributable to several potential mediators that may overlap in content (West \& Aiken, 1997). It is important to remember that a specific indirect effect through a mediator (say, $M_{3}$ ) in the multiple mediation context is not the same as the indirect effect through $M_{3}$ alone, except in the unlikely circumstance that all other mediators are uncorrelated with $M_{3}$. The specific indirect effect through $M_{3}$ represents the ability of $M_{3}$ to mediate the effect of $X$

\section{A}
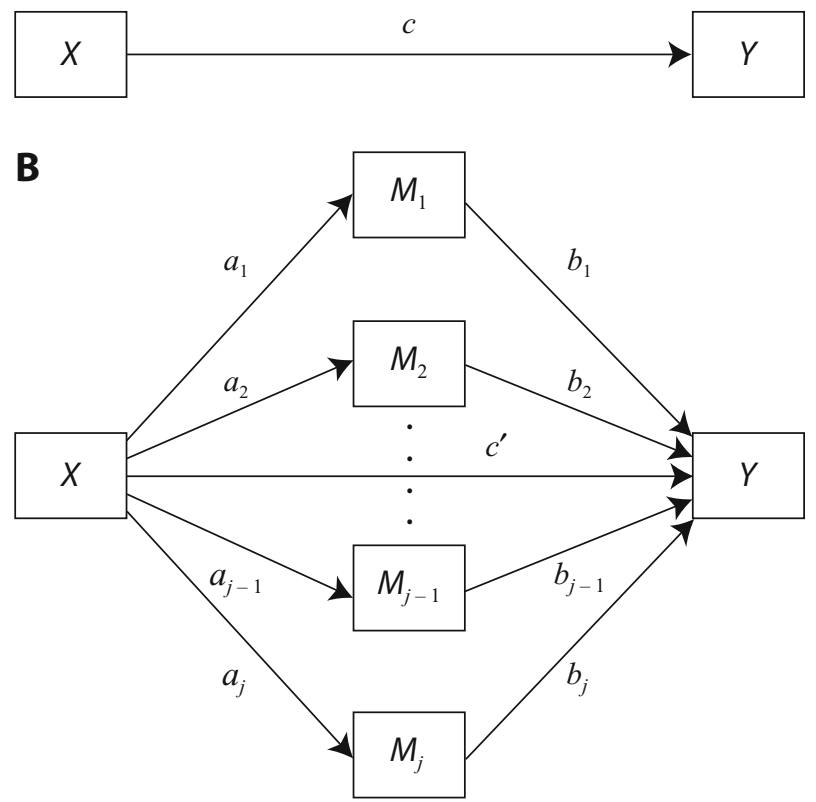

Figure 2. Illustration of a multiple mediation design with $j$ mediators. (A) $X$ affects $Y$. (B) $X$ is hypothesized to exert indirect effects on $Y$ through $M_{1}, M_{2}, \ldots, M_{j}$. 
on $Y$ conditional on the inclusion of the other mediators in the model. Because of this, collinearity plays a role in multiple mediation models in much the same way as in ordinary multiple regression. The effects of the mediators on $Y$ (the $b$ paths) are often attenuated to the degree to which the mediators are correlated, a phenomenon that can compromise the significance of particular specific indirect effects. In intervention research, for example, an intervention is sometimes designed to impact multiple intervening variables to achieve a desired outcome. In such cases, the mediators are almost necessarily correlated by virtue of their mutual reliance on a common cause, and individual mediators may not demonstrate large unique effects on the outcome.

We propose that investigating multiple mediation should involve two parts: (1) investigating the total indirect effect, or deciding whether the set of mediators transmits the effect of $X$ to $Y$; and (2) testing hypotheses regarding individual mediators in the context of a multiple mediator model (i.e., investigating the specific indirect effect associated with each putative mediator). We do not suggest that a significant total indirect effect is a prerequisite for investigating specific indirect effects. It is entirely possible to find specific indirect effects to be significant in the presence of a nonsignificant total indirect effect. ${ }^{2}$ Either or both types of effect may be of theoretical interest and worth investigating.

Several approaches have been suggested for assessing total and specific indirect effects in multiple mediator models. We focus on the four methods described earlier for testing a simple mediation hypothesis.

\section{Causal Steps Approach}

The causal steps approach can be used to determine whether or not $c-c^{\prime}$ represents a mediation effect in the multiple mediation context. Using this approach, the investigator asks whether the paths defining a specific indirect effect $\left(a_{i}\right.$ and $b_{i}$ in Figure 2) are significant. If either of the constituent paths for a hypothesized indirect effect through variable $M_{i}$ is not different from zero, then variable $M_{i}$ is deemed not to be a mediator of the effect of $X$ on $Y$. As noted earlier, the requirement that $c$ be significant is not always considered necessary for mediation to occur. It is possible, for example, for one variable $\left(M_{1}\right)$ to act as a mediator, and for a second $\left(M_{2}\right)$ to act as a suppressor (see MacKinnon et al., 2000). In effect, $M_{1}$ and $M_{2}$ may show indirect effects that cancel out.

Although it is easy to understand this extension of the causal steps approach, it suffers from the same shortcomings it has when used in the single mediator context, and for this reason we regard the causal steps approach of little utility in the multiple mediator context. It relies on a set of tests of individual $a$ and $b$ paths rather than testing the specific indirect effects, and yields no point estimate or $S E$ of the mediation effect.

\section{Product-of-Coefficients Approach}

A multivariate extension of the product-of-coefficients strategy is available for models involving multiple mediators. This test uses the multivariate delta method (Bishop,
Fienberg, \& Holland, 1975) to derive the $S E$ of the total indirect effect $\left(c-c^{\prime}\right)$. It is usually also of interest to investigate the specific indirect effects through individual mediators, which we discuss later. Because the mathematics are handled by the macros to be described later, we do not dwell on the derivations here but rather include them in supplementary material at the first author's Web site. ${ }^{3}$

The total indirect effect for a model including three mediators is simply the sum of the specific indirect effectsthat is, $\mathbf{f}=a_{1} b_{1}+a_{2} b_{2}+a_{3} b_{3}$. Using methods described by Bollen $(1987,1989)$, the asymptotic variance of a total indirect effect involving three mediators, for example, can be shown to equal:

$$
\begin{gathered}
\operatorname{var}[\mathbf{f}]=b_{1}^{2} s_{a_{1}}^{2}+a_{1}^{2} s_{b_{1}}^{2}+b_{2}^{2} s_{a_{2}}^{2}+a_{2}^{2} s_{b_{2}}^{2}+b_{3}^{2} s_{a_{3}}^{2}+a_{3}^{2} s_{b_{3}}^{2} \\
+2\left(a_{1} a_{2} s_{b_{1}, b_{2}}+a_{1} a_{3} s_{b_{1}, b_{3}}+a_{2} a_{3} s_{b_{2}, b_{3}}\right. \\
\left.+b_{1} b_{2} s_{a_{1}, a_{2}}+b_{1} b_{3} s_{a_{1}, a_{3}}+b_{2} b_{3} s_{a_{2}, a_{3}}\right),
\end{gathered}
$$

where subscripts indicate the mediator with which each of the $a$ and $b$ coefficients is associated. The square root of this quantity is the first-order $S E$ of the total indirect effect in a three-mediator model, assuming normality for the total indirect effect. A second-order version of the multivariate delta method can be employed to yield the exact $S E$ (see Preacher, Rucker, \& Hayes, 2007), although doing so improves accuracy only negligibly. MacKinnon (2000) provides a similar $S E$ for the test of the total indirect effect (involving four potential mediators), but his $S E$ assumes that the mediator residuals are uncorrelated, and consequently lacks terms containing covariances of the $a$ paths. If these parameters are free, the model is saturated (i.e., $d f=0$ ) and the $a$ path coefficients will typically have nonzero covariances. Regardless of which formula is used, the $S E$ for models involving fewer or more than four mediators can readily be obtained by either omitting terms from Equation 1 pertaining to nonexistent mediators in smaller models, or by adding terms pertaining to additional mediators in larger models. The formulae for specific indirect effects are the same as those for indirect effects in single-mediator models.

If path analysis or SEM is used to fit a multiple mediator model, as would typically be done, we recommend that residuals associated with the mediators be permitted to covary. There are examples in the literature where investigators fixed these residual covariances to zero (e.g., Holbert \& Stephenson, 2003; Rutter \& Hine, 2005), a practice that we do not endorse. Even if the mediator residual covariances are constrained to zero, the multiple mediation model still permits the mediators to covary to the extent that they mutually depend on $X$, so it is unclear what would be accomplished by constraining their residual covariances to zero. Doing so implies that the researcher hypothesizes that the covariances among the mediators are completely explained by their mutual dependence on $X$. This constraint may be difficult to defend on theoretical grounds, although it is a testable assumption. Second, residual correlations among mediators in such models can be substantial (e.g., Gudmundsdottir, Beck, 
Coffey, Miller, \& Palyo, 2004). If the mediator residuals are actually correlated and the researcher constrains the residual covariances to zero, the model will be misspecified; an unreasonable constraint will have been placed on the model. Model misspecification, in turn, can introduce substantial bias in SEs (Briggs, 2006; Cole \& Maxwell, 2003), although it will not bias point estimates of the $a$ or $b$ coefficients or the $S E$ s of the $a$ coefficients (in the models presented here). Under maximum likelihood estimation, the point estimates of all residual variances and coefficients will remain the same, whether or not the mediator residuals are permitted to covary. However, the $S E$ s of the $b$ paths will change, which in turn will affect normal-theory tests of total or specific indirect effects. Freeing residual covariances accounts for any unmodeled sources of covariation among mediators. In addition, whereas it is common to constrain the direct effect $\left(c^{\prime}\right)$ to zero in SEM-based tests of mediation, we recommend that $c^{\prime}$ be estimated instead. Constraining even small $c^{\prime}$ values potentially biases other path coefficients in the model, usually in a way that spuriously inflates estimates of indirect effects.

\section{Distribution of the Product Strategy}

Whereas the distribution of the product strategy can be used for testing specific indirect effects, the distribution of sums or differences of products (necessary for testing hypotheses about total indirect effects and pairwise contrasts among indirect effects, to be discussed later) has not yet been worked out. We regard this as an important area for future research.

\section{Bootstrapping}

One of the assumptions necessary for the use of $S E$ s derived via the delta method - therefore, a limitation of this multivariate extension of the product-of-coefficients strategy - is that of multivariate normality. Not only must the paths that constitute the indirect effects follow a multivariate normal distribution; it is further assumed that the sampling distributions of the total and specific indirect effects are normal. Sobel $(1982,1986)$ applied the delta method for this purpose with very large samples in mind, in which case it is reasonable to expect the sampling distribution to be approximately normal. However, in finite samples the total indirect effect is rarely normal, as we noted with respect to simple mediation earlier. To address this problem, Shrout and Bolger (2002) argued that bootstrapping methods can be extended to designs involving multiple mediation.

To bootstrap the sampling distribution of the specific and total indirect effects, take a sample of size $n$ cases with replacement from the original sample. In other words, a given case can be selected as part of a bootstrap sample not at all, once, twice, or even multiple times. Using this new resample of size $n$, reestimate all $j$ values of $a_{i}$ and $b_{i}$ and then calculate $a_{i} b_{i}^{*}$ and $\Sigma_{i}\left(a_{i} b_{i}^{*}\right)$, with the "**" denoting an estimate derived from the resampled data set. This process is repeated $k$ times, where $k$ is preferably at least 1,000 , yielding $k$ estimates of the total and specific indirect effects of $X$ on $Y$. The distributions of these $k$ estimates serve as empirical, nonparametric approximations of the sampling distributions of the indirect effects of interest. The bootstrap confidence interval (CI) for the populationspecific indirect effect through $M_{i}$ is derived by sorting the $k$ values of $a_{i} b_{i}^{*}$ from low to high. Values defining the lower and upper $100(\alpha / 2) \%$ of the distribution of $a_{i} b_{i}{ }^{*}$ are then found and taken as the lower and upper limits of the $100(1-\alpha) \%$ CI for the population indirect effect, where $\alpha$ is the desired nominal Type I error rate. More specifically, the lower and upper bounds of a $100(1-\alpha) \%$ CI are defined, respectively, as the $(.5 \alpha) k$ th and $1+(1-$ $.5 \alpha) k$ th values of $a_{i} b_{i}^{*}$ in this sorted distribution. For instance, $\alpha=.05$ generates a $95 \%$ CI. With $k=1,000$, the lower and upper bounds of the interval would be the 25 th and 976th values of $a_{i} b_{i}{ }^{*}$ in the sorted distribution of $a_{i} b_{i}{ }^{*}$. This procedure yields a percentile bootstrap CI. This same approach would be used to calculate a percentile bootstrap CI for the total indirect effect, replacing $a_{i} b_{i}^{*}$ in the discussion above with $\Sigma_{i}\left(a_{i} b_{i}^{*}\right)$.

Unlike regular CIs, percentile bootstrap CIs can be asymmetrical because they are based on an empirical estimation of the sampling distribution of the indirect effect, rather than on an assumption that the sampling distribution is normal. The sampling distribution of $a_{i} b_{i}^{*}$ is skewed relative to a normal distribution (unless $a=b=0$ ), and hence the confidence limits should not be equidistant from the point estimate. As Efron and Tibshirani (1993) suggest, the forced symmetry of ordinary CIs results in estimation inaccuracies and problems with Type I errors and power when used in hypothesis testing. Percentile bootstrap CIs can be improved by an adjustment to the percentile values of the sorted distribution of bootstrap estimates used for determining the bounds of the interval. For a technical and detailed treatment justifying the bias-corrected (BC) and bias-corrected and accelerated (BCa) intervals, see Efron (1987) and Efron and Tibshirani (1993).

To our knowledge, there are only two empirical investigations of the performance of bootstrapping in a multiplemediator context. Briggs (2006) examined the accuracy of percentile, $\mathrm{BC}$, and $\mathrm{BCa}$ CIs for estimating total and specific indirect effects in a two-mediator model, comparing their performance to the product-of-coefficients strategy, both in terms of coverage of the known effect and in hypothesis testing when sampling from 16 different populations exhibiting various degrees of mediation. She found that bootstrapping generally was superior to the multivariate product-of-coefficients strategy in small to moderate samples, with $\mathrm{BC}$ and $\mathrm{BCa}$ performing best in terms of both power and Type I error rates. Williams (2004) and Williams and MacKinnon (2008) compared the product-of-coefficients strategy to percentile and $\mathrm{BC}$ methods, examining both Type I error rates and power in a three-mediator model with two outcomes. Bootstrapping generally was superior to the product-of-coefficients strategy. There were differences between the percentile and bias corrected methods, depending on the size of the paths in the model, with the percentile method generally showing a slight superiority in conditions in which the bias corrected method was slightly liberal. As bootstrapping becomes more widely known and implemented, we 
will no doubt see more research comparing the methods. For now, the evidence supports our claim that the bootstrapping methods we describe here are preferred over methods that assume symmetry or normality of the sampling distribution of the indirect effect.

Earlier we noted that the choice between freely estimating or constraining the mediator residual covariances to zero can affect the validity of inferences, using the product-of-coefficients approach. Not so with bootstrapping. Because point estimates of path coefficients do not depend on the choice to free or constrain residual covariances, bootstrap confidence intervals do not depend on this choice.

\section{CONTRASTING INDIRECT EFFECTS IN MULTIPLE MEDIATOR MODELS}

It is sometimes important to test the hypothesis that two indirect effects - whatever their magnitudes may be - are equal in size. For example, it may be of interest to compare competing theories about the mode by which attitudes affect behavior by including two or more mediators in one model and then comparing the strengths of the two indirect effects to decide which theory should be given more credence. Other occasions may demand more complex contrasts. For example, it may be important to test the hypothesis that the size of one indirect effect is different from the average size of two other indirect effects linking the same pair of independent and dependent variables. Researchers conducting contrasts should keep in mind that contrasts do not compare indirect effects per se, but rather specific indirect effects - the unique abilities of each mediator to account for the effect of $X$ on $Y$. Contrasts represent comparisons of indirect effects only insofar as the mediators are themselves uncorrelated.

MacKinnon (2000) provides the only statistical treatment of contrast hypotheses such as these. Of central importance is his observation that all mediators of the same $X \rightarrow Y$ effect are quantified in the same metric - that of the dependent variable. To see why this is so, first note that any regression coefficient can be expressed as a function of a term involving only correlations and a term involving the scales (standard deviations, $S D \mathrm{~s}$ ) of the two variables involved. In the case of a single mediator (say, $M_{1}$ ) in a two-mediator model,

$$
a_{1}=r_{X M_{1}} \frac{S D_{M_{1}}}{S D_{X}}
$$

and Equation 3 below (Cohen, Cohen, West, \& Aiken, 2003, Appendix 2). When $a_{1}$ and $b_{1}$ are multiplied together, $S D_{M_{1}}$ cancels out, leaving a result that is free of the metric of $M_{1}$. This observation justifies the comparison of multiple indirect effects linking the same independent-dependent vari- able pair. The product-of-coefficients and bootstrapping methods may be used to test hypotheses about contrasts, just as with total and specific indirect effects.

Consider first the case in which the researcher wishes to contrast the indirect effects through mediators $M_{1}$ and $M_{2}$. The value of the contrast is

$$
\mathbf{f}_{c}=a_{1} b_{1}-a_{2} b_{2} .
$$

The delta method yields

$$
\begin{aligned}
\operatorname{var}\left[\mathbf{f}_{c}\right]= & b_{1}^{2} \sigma_{a_{1}}^{2}-2 b_{1} b_{2} \sigma_{a_{1}, a_{2}}+b_{2}^{2} \sigma_{a_{2}}^{2} \\
& +a_{1}^{2} \sigma_{b_{1}}^{2}-2 a_{1} a_{2} \sigma_{b_{1}, b_{2}}+a_{2}^{2} \sigma_{b_{2}}^{2}
\end{aligned}
$$

in the case where residuals among mediators are allowed to covary (see Bollen, 1987, 1989). Constraining residual covariances to zero results in independent $a$ paths, yielding the formula derived by MacKinnon (2000) for the variance of a single pairwise contrast,

$$
\begin{aligned}
\operatorname{var}\left[\mathbf{f}_{c}\right]= & b_{1}^{2} \sigma_{a_{1}}^{2}+b_{2}^{2} \sigma_{a_{2}}^{2}+a_{1}^{2} \sigma_{b_{1}}^{2} \\
& -2 a_{1} a_{2} \sigma_{b_{1}, b_{2}}+a_{2}^{2} \sigma_{b_{2}}^{2} .
\end{aligned}
$$

As with specific and total indirect effects, the square root of this variance is the first-order $S E$ of the contrast, assuming normality for the contrast, and can thus be used to test hypotheses or to construct CIs. MacKinnon (2000) discusses several other useful contrasts and derives $S E$ s for them under the assumption that the mediator residual covariances are constrained to zero. Any contrasts may be specified and tested using bootstrapping in lieu of deriving normal-theory $S E$ s using the same logic described above. The contrast is computed after the manner of Equation 4 for each bootstrap resample, and a sampling distribution of this contrast is generated. The $100(\alpha / 2)$ th and $100(1-\alpha / 2)$ th percentiles (or bias-corrected versions) are then used to test the hypothesis that the contrast equals zero. Because point estimates of path coefficients do not depend on the choice to free or constrain residual covariances, bootstrap intervals do not depend on this choice.

\section{SOFTWARE IMPLEMENTATION}

With the exception of the causal steps strategy, a procedure not well suited to the multiple mediator case, the procedures described here are computationally tedious and impractical without the aid of a computer. Fortunately, several SEM programs can conduct hypothesis tests and construct CIs for indirect effects in both simple and multiple mediator models, using either the product-of-coefficients strategy or bootstrapping in some form. AMOS (Arbuckle, 1999) implements the percentile bootstrap method for total indirect effects in simple and multiple mediator models. Mplus (Muthén \& Muthén, 2007) is the only program of

$$
b_{1}=\frac{\left(r_{X M_{2}} r_{M_{1} M_{2}}-r_{X M_{1}}\right) r_{Y X}+\left(1-r_{X M_{2}}^{2}\right) r_{Y M_{1}}+\left(r_{X M_{1}} r_{X M_{2}}-r_{M_{1} M_{2}}\right) r_{Y M_{2}}}{1-r_{X M_{1}}^{2}-r_{X M_{2}}^{2}-r_{M_{1} M_{2}}^{2}+2 r_{X M_{1}} r_{X M_{2}} r_{M_{1} M_{2}}}\left(\frac{S D_{Y}}{S D_{M_{1}}}\right)
$$


which we know that implements both normal-theory and bootstrap methods (percentile and BC) for estimating both total and specific indirect effects. Additionally, it is possible to program Mx (Neale, Boker, Xie, \& Maes, 2003) to supply bootstrap intervals for virtually any function of model parameters. Some programs, such as LISREL and Mplus, can be programmed to conduct contrasts of indirect effects (Cheung, 2007). We include Mplus syntax in Appendix A for estimating and contrasting specific indirect effects in a generic two-mediator model using $\mathrm{BC}$ bootstrapping. It is also worth mentioning that some SEM programs can be used to impose nonlinear constraints (e.g., contrasts of indirect effects), with the resulting lack of fit reflected by a change in the $\chi^{2}$ fit statistic. The performance of this likelihood ratio (LR) test for contrasts of indirect effects has not been formally examined in simulation studies, but in our experience tends to yield results similar to tests using a delta method $S E$. We do not examine this method here, but LISREL syntax is included in Appendix A for contrasting indirect effects using the LR test.

Mediation models are best estimated in a SEM context because of the greater flexibility SEM programs afford in model specification and estimation options. Although specialized SEM software is being used with increasing frequency to test mediation hypotheses, many researchers still rely on historically popular programs, such as SPSS or SAS, for their analyses. Neither SPSS nor SAS has the intrinsic capability to conduct the type of analysis we have described here, but both have built-in programming languages that allow users to create customized data analysis routines. We have developed macros for SPSS and SAS that provide researchers who prefer these programs the ability to conduct the type of analysis we have described here. The user can request tests of total and specific indirect effects using the product-of-coefficients approach or by bootstrapping confidence intervals (percentile, $\mathrm{BC}$, and $\mathrm{BCa}$ ) at any desired confidence level (Mplus is inflexible in this regard). Additionally, at the user's request, the macro conducts all possible pairwise contrasts of indirect effects in multiple mediator models. It also allows for statistical control of one or more covariates that are not proposed to be mediators of the total effect. The length of the macros precludes publication here, but they may be downloaded free from www .quantpsy.org, along with documentation. We now provide an illustrative example using the product-of-coefficients strategy (which assumes a large sample) and the SPSS version of the macro for bootstrap results.

\section{ILLUSTRATIVE EXAMPLE}

Klein, Fan, and Preacher (2006) tested several hypotheses linking the early socialization experiences of new employees - specifically, the realism of preentry knowledge (the quality and quantity of job-related information gained by new employees prior to entering employment) and the perceived helpfulness of socialization agents - to socialization outcomes, including job satisfaction, affective organizational commitment, and job role clarity. Several dimensions of socialization content mastery were proposed as putative mediators for these relationships. Five of the dimensions identified by Chao, O'Leary-Kelly, Wolf, Klein, and Gardner (1994) — organizational goals and values, people, history, job performance proficiency, and politics - were considered. The basic theory underlying the hypotheses was that early socialization experiences are related to socialization outcomes, through their impact on the mastery of various facets of organizational socialization. However, not all socialization content dimensions were hypothesized as mediators for all relationships.

For illustrative purposes, we focus on a subset of hypotheses linking the helpfulness of socialization agents (helpful) to future job satisfaction (jobsat). Agent helpfulness reflects the extent to which more senior coworkers, supervisors, and administrative assistants were available and helpful when a new employee started at a new job. Klein et al. (2006) hypothesized that the mastery of three of the above five socialization content dimensions would mediate the effect of agent helpfulness on job satisfaction. Those dimensions were job performance proficiency ( perform, the employee's understanding of the tasks that need to be performed and how to perform them), people (people, the establishment of successful work relationships), and politics (politics, the understanding of both formal and informal work relationships and power structures). Whereas three mediators were hypothesized, Klein et al. found the predicted indirect effect only for the people dimension. The analyses discussed here differ from those conducted by Klein et al. in important ways. First, no latent variables are employed; rather, for reasons of pedagogical simplicity, the indicators of the latent variables used for the three socialization content dimensions and for job satisfaction will be condensed to single measured variables. We use a subsample of 141 individuals with complete data on the variables of interest. Furthermore, in Klein et al., no model involving more than one mediator was assessed.

The total indirect effect of $X$ on $Y$ is $\mathbf{f}=a_{1} b_{1}+a_{2} b_{2}+$ $a_{3} b_{3}=.1074$. To determine the significance of this effect, we employ Equation 1, and find that the asymptotic variance is $\operatorname{var}[\mathbf{f}]=.0009719$. The asymptotic critical ratio for the total indirect effect of $X$ on $Y$ is therefore

$$
Z=\frac{.1074}{\sqrt{.0009719}}=3.445,
$$

which leads to a rejection of the null hypothesis that the total indirect effect is zero $(p=.0006)$.

In multiple mediation models, the researcher is concerned not only with the total indirect effect of $X$ on $Y$, but also with specific indirect effects. The specific indirect effects are $a_{1} b_{1}=.0222$ (through politics), $a_{2} b_{2}=.0796$ (through people), and $a_{3} b_{3}=.0057$ (through perform). The $S E$ s and critical ratios for these effects are reported in Table 1. Of the potential mediators examined, we can conclude that people is likely an important mediator $(Z=$ $2.94, p=.0033$ ). Confidence intervals for each indirect effect can be computed in the usual way.

Because the assumption of normality of the sampling distribution of the total and specific indirect effects is questionable, particularly in small samples, as in our il- 
Table 1

Mediation of the Effect of Perceived Helpfulness of Existing Employees on Job Satisfaction Through New Employees' Understanding of Performance Standards, Formation of Social Networks, and Organization Politics

\begin{tabular}{|c|c|c|c|c|c|c|c|c|c|}
\hline & \multirow{3}{*}{$\begin{array}{c}\text { Point } \\
\text { Estimate }\end{array}$} & \multirow{2}{*}{\multicolumn{2}{|c|}{$\begin{array}{c}\text { Product of } \\
\text { Coefficients }\end{array}$}} & \multicolumn{6}{|c|}{ Bootstrapping } \\
\hline & & & & \multicolumn{2}{|c|}{ Percentile 95\% CI } & \multicolumn{2}{|c|}{$\mathrm{BC} 95 \% \mathrm{CI}$} & \multicolumn{2}{|c|}{$\mathrm{BCa} 95 \% \mathrm{CI}$} \\
\hline & & $S E$ & $Z$ & Lower & Upper & Lower & Upper & Lower & Upper \\
\hline Politics & 0.0222 & 0.0210 & 1.0555 & -0.0173 & 0.0677 & -0.0151 & 0.0707 & -0.0164 & 0.0691 \\
\hline People & 0.0796 & 0.0271 & 2.9366 & 0.0312 & 0.1394 & 0.0332 & 0.1430 & 0.0347 & 0.1481 \\
\hline \multicolumn{10}{|c|}{ Contrasts } \\
\hline Politics vs. perform & 0.0165 & 0.0284 & 0.5801 & -0.0328 & 0.0684 & -0.0331 & 0.0684 & -0.0328 & 0.0687 \\
\hline People vs. perform & 0.0739 & 0.0325 & 2.2730 & 0.0104 & 0.1498 & 0.0130 & 0.1523 & 0.0167 & 0.1576 \\
\hline
\end{tabular}

Note-BC, bias corrected; BCa, bias corrected and accelerated; 5,000 bootstrap samples.

lustration, we bootstrapped the indirect effects of helpful on jobsat, using the SPSS version of our macro. The estimates and $95 \% \mathrm{CIs}$ (percentile, $\mathrm{BC}$, and $\mathrm{BCa}$ ) are in Table 1. In agreement with results of the product-ofcoefficients strategy, people is the only significant mediator of the helpful $\rightarrow$ jobsat relationship.

To briefly illustrate how a pairwise contrast of two indirect effects may be conducted, consider two of the indirect effects examined in our running example, through politics and people. It was found earlier that the specific indirect effect of helpful on jobsat through politics was not significantly different from zero, but that the specific indirect effect through people was significant. It may be of interest to see whether these two indirect effects differ significantly. Using Equation 4 to define the contrast and Equation 5 to define its sampling variance yields $\mathbf{f}_{c}=$ $(0.2106)(0.1052)-(0.2281)(0.3489)=-0.0574$ and $\operatorname{var}\left[\mathbf{f}_{c}\right]=0.001269$. A $95 \% \mathrm{CI}$ for the contrast is therefore $\left\{-.0574 \pm 1.96(.001269)^{1 / 2}\right\}$, or $\{-.127, .012\}$. Because zero is contained in the interval, the two indirect effects cannot be distinguished in terms of magnitude, despite the fact that one is significantly different from zero and the other is not. Such apparent paradoxes can occur when one of the specific indirect effects involved in the contrast is not sufficiently far from zero. ${ }^{4}$

Appendix B contains the full macro output. The bootstrap estimates presented here are based on 5,000 bootstrap samples. ${ }^{5}$ The interpretation of these results is that, taken as a set, people, politics, and performance do mediate the effect of helpful on jobsat. As can be seen in Appendix B, the total and direct effects of helpful on jobsat are $0.2131, p<.01$, and $0.1057, p<.07$, respectively. The difference between the total and direct effects is the total indirect effect through the three mediators, with a point estimate of .1074 and a 95\% BCa bootstrap CI of 0.0612 to 0.1680 (i.e., we can claim that the difference between the total and the direct effect of helpful on jobsat is different from zero). From output in Appendix B, the directions of the $a$ and $b$ paths are consistent with the interpretation that greater helpfulness leads to greater mastery of the politics, people, and performance dimensions, which in turn leads to greater job satisfaction. An examination of the specific indirect effects indicates that only people is a mediator, since its $95 \%$ CI does not contain zero. Neither politics nor performance contributes to the indirect effect above and beyond people. Examination of the pairwise contrasts of the indirect effects $(\mathrm{C} 1$, $\mathrm{C} 2$, and $\mathrm{C} 3$ in Appendix B) shows that the specific indirect effect through people is larger than the specific indirect effect through performance, with a BCa $95 \%$ CI of 0.0167 to 0.1576 . Note that a formal $p$-value is provided for these effects in the section of output labeled "Normal Theory Tests for Indirect Effects," but these tests assume the sampling distribution of the indirect effect (or their differences, in the case of pairwise contrasts) is normal, an assumption unnecessary for valid inference when bootstrapping is used. Observe that this interpretation of the mediation analysis does not focus at all on the statistical significance of the $a$ and $b$ paths, as is required using the causal steps method. Instead, emphasis is placed almost entirely on the direction and size of the indirect effects.

\section{DISCUSSION}

\section{Recommendations}

When the hypothesis of mediation by multiple potential mediators is entertained, multiple mediation is the appropriate analytic strategy. We have presented and discussed ways by which hypotheses of multiple mediation may be evaluated. The product-of-coefficients strategy is useful only when the assumption of normality of the sampling distribution of the indirect effect can be reasonably met, as when large samples are available or when the effects are large.

Bootstrapping provides the most powerful and reasonable method of obtaining confidence limits for specific indirect effects under most conditions, so our primary recommendation is to use bootstrapping - in particular, BC bootstrapping - whenever possible; see Briggs (2006), Williams (2004), and Williams and MacKinnon (2008) for extensive simulation results supporting this recommendation. We also recommend that researchers entertain the possibility of multiple mediators. In most situations, it is unlikely that the effect of an independent variable on an outcome is transmitted by only one means. When multiple mediators are entertained, it is often more 
convenient, precise, and parsimonious to include all of them in the same model.

Potential mediators should be selected on the basis of theory. A specific indirect effect does not represent the ability of a given mediator $M$ to mediate the effect of $X$ on $Y$. Rather, a specific indirect effect represents the ability of $M$ to mediate the effect controlling for all other mediators. Thus, a specific indirect effect for $M_{2}$ represents $M_{2}$ 's unique ability to mediate the $X \rightarrow Y$ relationship. Specific indirect effects will generally, but not necessarily, be attenuated, to the extent that the mediators are correlated. This is simply a manifestation of the common phenomenon of collinearity, or redundancy among predictors (mediators are predictors of $Y$ ). Collinearity is not necessarily a problem, but it may lead the investigator to conclude that $M$ does not serve as a mediator when in fact it does, or even to conclude that $M$ serves as a mediator when it does not. Another consequence of the fact that mediators are usually correlated is that contrasts do not compare two mediators in their ability to mediate, but rather their unique abilities to mediate, above and beyond any other mediators or covariates in the model. Researchers may wish to consider these facts when selecting mediators for inclusion in a multiple mediator model. In situations permitting such control, it will generally be to the researcher's advantage to select mediators that represent unique constructs with as little conceptual overlap as possible. Following this strategy will minimize collinearity.

\section{Extensions}

Despite conceptual distinctions, mediation, confounding, and suppression effects share a common mathematical basis (MacKinnon et al., 2000). Therefore, similar methods may be used to investigate each of these effects. As mentioned earlier, it is possible for several mediators and suppressors to coexist in models involving multiple intervening variables (MacKinnon et al., 2000). Under such circumstances, tests of specific indirect effects, whether by using the product-of-coefficients approach or the resampling approach, would facilitate teasing apart the separate roles played by individual intervening variables.

The examples presented in this article involved only measured variables, with no correction for unreliability. It should be emphasized that the results generalize to SEM with latent variables. One of the most attractive features of SEM is that, unlike regression analysis of the type performed with SAS or SPSS, it explicitly models measurement error, allowing researchers to test hypotheses using the latent constructs rather than imperfect measured indicators. Many SEM packages now include delta method estimates of $S E$ s for indirect effects. Macros are provided for bootstrapping confidence limits for simple mediation in EQS and AMOS (Shrout \& Bolger, 2002), and LISREL, Mplus, and Mx can generate bootstrap limits for total or specific indirect effects in the multiple mediation context. Our SPSS and SAS macros can be used for multiple mediation in the SEM special case of path analysis, in which all variables are observed.
Finally, the macros presented here may be applied and extended in creative ways. For example, the ability to include covariates permits the testing of mediated moderation effects (Baron \& Kenny, 1986), in which interaction effects are hypothesized to be mediated; future research might also address how mediated moderation effects may be contrasted in a pairwise manner. The macros can also be used to estimate indirect effects in models with $k$ independent variables by estimating $k$ models. In each of these models, one of the variables is chosen as the independent variable, and the remaining $k-1$ variables are treated as covariates. Future work could also address methods for investigating simple and multiple mediation in models involving multiple outcome measures, nonlinear effects, multilevel designs, or missing data.

\section{AUTHOR NOTE}

This work was funded in part by National Institute on Drug Abuse Grant DA16883, awarded to K.J.P. while at the University of North Carolina at Chapel Hill. We thank Howard Klein for permission to use illustrative data and for providing comments, and Kenneth Bollen and Jinyan Fan for providing helpful feedback. The SPSS and SAS macro command set is available online through www.quantpsy.org or by contacting the authors. Correspondence should be addressed to K. J. Preacher, Department of Psychology, University of Kansas, 1415 Jayhawk Blvd., Rm. 426, Lawrence, KS 66045-7556 (e-mail: preacher@ku.edu).

\section{REFERENCES}

Aiken, L. S., West, S. G., Woodward, C. K., Reno, R. R., \& ReynOLDS, K. D. (1994). Increasing screening mammography in asymptomatic women: Evaluation of a second-generation theory-based program. Health Psychology, 13, 526-538.

ARBuCKLE, J. L. (1999). AMOS 4 [Computer software]. Chicago: SmallWaters Corp.

Aroian, L. A. (1947). The probability function of the product of two normally distributed variables. Annals of Mathematical Statistics, 18, 265-271.

Assad, K. K., Donnellan, M. B., \& Conger, R. D. (2007). Optimism: An enduring resource for romantic relationships. Journal of Personality \& Social Psychology, 93, 285-297.

AzEn, R. (2003, August). Multiple mediator models: A comparison of testing approaches. Poster presented at the 111th Annual Convention of the American Psychological Association, Toronto, Ontario.

BARON, R. M., \& KenNy, D. A. (1986). The moderator-mediator variable distinction in social psychological research: Conceptual, strategic, and statistical considerations. Journal of Personality \& Social Psychology, 51, 1173-1182.

Bishop, Y. M., Fienberg, S. E., \& Holland, P. W. (1975). Discrete multivariate analysis: Theory and practice. Cambridge, MA: MIT Press.

Bollen, K. A. (1987). Total, direct, and indirect effects in structural equation models. Sociological Methodology, 17, 37-69.

Bollen, K. A. (1989). Structural equations with latent variables. New York: Wiley.

Bollen, K. A., \& Stine, R. (1990). Direct and indirect effects: Classical and bootstrap estimates of variability. Sociological Methodology, 20, $115-140$.

BRIGGS, N. (2006). Estimation of the standard error and confidence interval of the indirect effect in multiple mediator models. Dissertation Abstracts International, 37, 4755B.

Brown, R. L. (1997). Assessing specific mediational effects in complex theoretical models. Structural Equation Modeling, 4, 142-156.

Carson, J. W., Carson, K. M., Gil, K. M., \& Baucom, D. H. (2007). Self-expansion as a mediator of relationship improvements in a mindfulness intervention. Journal of Marital \& Family Therapy, 33, 517-528.

Chao, G. T., O'Leary-Kelly, A. M., Wolf, S., Klein, H. J., \& GardNER, P. D. (1994). Organizational socialization: Its content and consequences. Journal of Applied Psychology, 79, 730-743. 
Cheung, M. W.-L. (2007). Comparison of approaches to constructing confidence intervals for mediating effects using structural equation models. Structural Equation Modeling, 14, 227-246.

Cohen, J., Cohen, P., West, S. G., \& Aiken, L. S. (2003). Applied multiple regression/correlation analysis for the behavioral sciences (3rd ed.). Mahwah, NJ: Erlbaum.

Cole, D. A., \& MaXwell, S. E. (2003). Testing mediational models with longitudinal data: Questions and tips in the use of structural equation modeling. Journal of Abnormal Psychology, 112, 558-577.

Collins, L. M., Graham, J. W., \& Flaherty, B. P. (1998). An alternative framework for defining mediation. Multivariate Behavioral Research, 33, 295-312.

Constantine, M. G. (2007). Racial microaggressions against African American clients in cross-racial counseling relationships. Journal of Counseling Psychology, 54, 1-16.

Craig, C. C. (1936). On the frequency function of xy. The Annals of Mathematical Statistics, 7, 1-15.

DE Zavala, A. G., \& VAn Bergh, A. (2007). Need for cognitive closure and conservative political beliefs: Differential mediation by personal worldviews. Political Psychology, 28, 587-608

EFron, B. (1987). Better bootstrap confidence intervals. Journal of the American Statistical Association, 82, 171-185.

EFron, B., \& Tibshirani, R. J. (1993). An introduction to the bootstrap. Boca Raton, FL: Chapman \& Hall.

Fox, J. (1985). Effects analysis in structural equation models II: Calculation of specific indirect effects. Sociological Methods \& Research, 14, 81-95.

Gudmundsdottir, B., Beck, J. G., Coffey, S. F., Miller, L., \& Palyo, S. A. (2004). Quality of life and post trauma symptomatology in motor vehicle accident survivors: The mediating effects of depression and anxiety. Depression \& Anxiety, 20, 187-189.

Holbert, R. L., Shah, D. V., \& KwaK, N. (2003). Political implications of prime-time drama and sitcom use: Genres of representation and opinions concerning women's rights. Journal of Communication, $\mathbf{5 3}, 45-60$.

Holbert, R. L., \& Stephenson, M. T. (2003). The importance of indirect effects in media effects research: Testing for mediation in structural equation modeling. Journal of Broadcasting \& Electronic Media, 47, 556-572.

Judd, C. M., \& Kenny, D. A. (1981). Process analysis: Estimating mediation in treatment evaluations. Evaluation Review, 5, 602-619.

Kalyanaraman, S., \& Sundar, S. S. (2006). The psychological appeal of personalized content in web portals: Does customization affect attitudes and behavior? Journal of Communication, 56, 110-132.

Kenny, D. A., Kashy, D. A., \& Bolger, N. (1998). Data analysis in social psychology. In D. Gilbert, S. T. Fiske, \& G. Lindzey (Eds.), Handbook of social psychology (4th ed., Vol. 1, pp. 233-265). New York: McGraw-Hill

Klein, H. J., Fan, J., \& Preacher, K. J. (2006). The effects of early socialization experiences on content mastery and outcomes: A mediational approach. Journal of Vocational Behavior, 68, 96-115.

Lockwood, C. M., \& MacKinnon, D. P. (1998). Bootstrapping the standard error of the mediated effect. Proceedings of the 23rd Annual Meeting of SAS Users Group International (pp. 997-1002). Cary, NC: SAS Institute, Inc.

MaCKINNON, D. P. (1994). Analysis of mediating variables in prevention and intervention research. In A. Cázares \& L. Beatty (Eds.), Scientific methods for prevention intervention research (NIDA Monograph No. 139, pp. 127-153). Rockville, MD: National Institute on Drug Abuse.

MacKinnon, D. P. (2000). Contrasts in multiple mediator models. In J. Rose, L. Chassin, C. C. Presson, \& S. J. Sherman (Eds.), Multivariate applications in substance use research: New methods for new questions (pp. 141-160). Mahwah, NJ: Erlbaum.

MacKinnon, D. P., \& DwYER, J. H. (1993). Estimating mediated effects in prevention studies. Evaluation Review, 17, 144-158.

MacKinnon, D. P., Fritz, M. S., Williams, J., \& Lockwood, C. M. (2007). Distribution of the product confidence limits for the indirect effect: Program PRODCLIN. Behavior Research Methods, 39, 384-389.

MacKinnon, D. P., Krull, J. L., \& Lockwood, C. M. (2000). Equivalence of the mediation, confounding, and suppression effect. Prevention Science, 1, 173-181.
MacKinnon, D. P., Lockwood, C. M., Hoffman, J. M., West, S. G., \& SheETs, V. (2002). A comparison of methods to test mediation and other intervening variable effects. Psychological Methods, 7, 83-104.

MacKinnon, D. P., Lockwood, C. M., \& Williams, J. (2004). Confidence limits for the indirect effect: Distribution of the product and resampling methods. Multivariate Behavioral Research, 39, 99-128.

Morgeson, F. P., Delaney-Klinger, K., \& Hemingway, M. A. (2005) The importance of job autonomy, cognitive ability, and job-related skill for predicting role breadth and job performance. Journal of Applied Psychology, 90, 399-406.

Muthén, L. K., \& MuthéN, B. O. (2007). Mplus user's guide (5th ed.). Los Angeles: Muthén \& Muthén.

Neale, M. C., BoKer, S. M., XIe, G., \& Maes, H. H. (2003). Mx: Statistical modeling (6th ed.). Richmond, VA: Department of Psychiatry, Virginia Commonwealth University.

Preacher, K. J., \& Hayes, A. F. (2004). SPSS and SAS procedures for estimating indirect effects in simple mediation models. Behavior Research Methods, Instruments, \& Computers, 36, 717-731.

Preacher, K. J., \& HaYes, A. F. (2008). Contemporary approaches to assessing mediation in communication research. In A. F. Hayes, M. D. Slater, \& L. B. Snyder (Eds.), The Sage sourcebook of advanced data analysis methods for communication research (pp. 13-54). Thousand Oaks, CA: Sage.

Preacher, K. J., Rucker, D. D., \& Hayes, A. F. (2007). Assessing moderated mediation hypotheses: Theory, methods, and prescriptions. Multivariate Behavioral Research, 42, 185-227.

Raver, J. L., \& Gelfand, M. J. (2005). Beyond the individual victim: Linking sexual harassment, team processes, and team performance. Academy of Management Journal, 48, 387-400.

Reynolds, K. D., Bishop, D. B., Chou, C.-P., Xie, B., Nebeling, L., \& Perry, C. L. (2004). Contrasting mediating variables in two 5-a-day nutrition intervention programs. Preventive Medicine, 39, 882-893.

RutTer, A., \& Hine, D. W. (2005). Sex differences in workplace aggression: An investigation of moderation and mediation effects. Aggressive Behavior, 31, 254-270.

Shrout, P. E., \& Bolger, N. (2002). Mediation in experimental and nonexperimental studies: New procedures and recommendations. Psychological Methods, 7, 422-445.

Sobel, M. E. (1982). Asymptotic confidence intervals for indirect effects in structural equations models. In S. Leinhart (Ed.), Sociological methodology 1982 (pp. 290-312). San Francisco: Jossey-Bass.

SoBEL, M. E. (1986). Some new results on indirect effects and their standard errors in covariance structure models. In N. Tuma (Ed.), Sociological Methodology 1986 (pp. 159-186). Washington, DC: American Sociological Association.

SPRINGER, M. D. (1979). The algebra of random variables. New York: Wiley.

TaYlor, A. B., MacKinnon, D. P., \& Tein, J.-Y. (2008). Tests of the three-path mediated effect. Organizational Research Methods, 11, 241-269.

West, S. G., \& AiKEN, L. S. (1997). Toward understanding individual effects in multicomponent prevention programs: Design and analysis strategies. In K. J. Bryant, M. Windle, \& S. G. West (Eds.), The science of prevention: Methodological advances from alcohol and substance abuse research (pp. 167-209). Washington, DC: American Psychological Association.

WiLLIAMS, J. (2004). Resampling and distribution of the product methods for testing indirect effects in complex models. Unpublished doctoral dissertation, Arizona State University, Tempe, AZ.

Williams, J., \& MacKinnon, D. P. (2008). Resampling and distribution of the product methods for testing indirect effects in complex models Structural Equation Modeling, 15, 23-51.

\section{NOTES}

1. Other forms of mediation are possible, such as models of the form $X \rightarrow M_{1} \rightarrow M_{2} \rightarrow Y$. For a discussion of such models, see Taylor, MacKinnon, and Tein (2008)

2. This pattern may occur in models containing both a mediation effect and a suppression effect. Even though either effect may be large and significant, their sum may be small and nonsignificant.

3. www.quantpsy.org. 
4. Such paradoxes occur frequently and are a result, in part, of thinking about effects in dichotomous terms - as zero or something else. For example, a researcher may reject an omnibus null hypothesis that three means are different from each other using ANOVA even though post hoc comparisons between means may fail to find that any two means are statistically different. Or the multiple correlation in a regression analysis may be different from zero even though none of the regression coefficients for the individual predictors are. Ultimately, such paradoxes result from conducting multiple tests that differ in power.
5. There is no consensus as to how many bootstrap samples should be generated, except that more is better. Given that the percentile endpoints are estimated using extremes of the sampling distribution and that, for $\mathrm{BCa}$ intervals, a good estimate of the acceleration constant is needed, it is important to minimize sampling variance in these estimates. We recommend at least 5,000 resamples for final reporting, although 1,000 is probably sufficient for preliminary analyses. There is little reason to use fewer, given the speed of today's desktop computing technology.

\section{APPENDIXA \\ Mplus and LISREL Syntax}

The following Mplus syntax assumes that the user has a data set with four variables (x, $\mathrm{m} 1, \mathrm{~m} 2$, and $\mathrm{y}$ ) and that it is of interest to estimate the specific indirect effects of $\mathrm{x}$ on $\mathrm{y}$ through $\mathrm{m} 1$ and $\mathrm{m} 2$ using bias-corrected bootstrapping. If it is desired to test the contrast hypothesis that the two indirect effects are equal, the "!" symbols should be omitted before running.

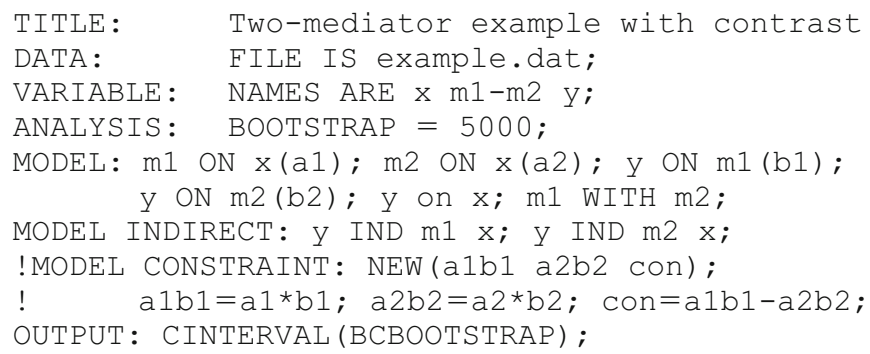

The following LISREL syntax assumes that the user has a data set with four variables $(\mathrm{x}, \mathrm{m} 1, \mathrm{~m} 2$, and $\mathrm{y} ; N=$ 20 ) and that it is of interest to contrast the two indirect effects using a likelihood ratio test. The $p$-value associated with the reported $\chi^{2}$ statistic reflects the result of the LR test.

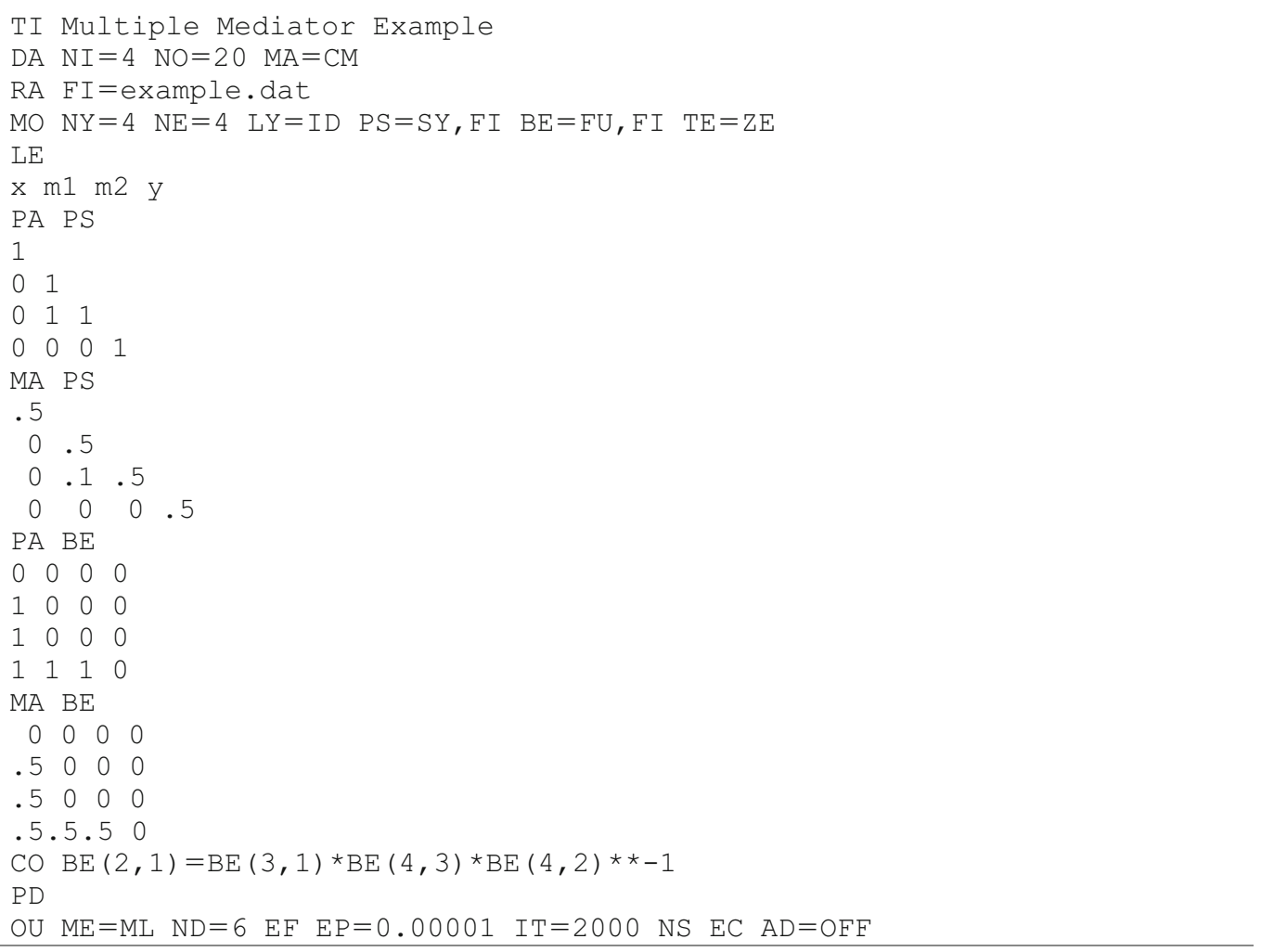


APPENDIX B

SPSS Macro Input and Output

The macro command set (available for both SPSS and SAS) can be found at www.quantpsy.org.

Macro command line:

INDIRECT $\mathrm{Y}=$ jobsat $/ \mathrm{X}=$ helpful/M = politics people perform $/ \mathrm{C}=0 / \mathrm{BOOT}=$ $5000 / \mathrm{CONF}=95 / \mathrm{CONTRAST}=1 / \mathrm{PERCENT}=1 / \mathrm{BC}=1 / \mathrm{BCA}=1$.

Macro output:

Run MATRIX procedure:

Dependent, Independent, and Proposed Mediator Variables:

$\mathrm{DV}=$ jobsat

IV $=$ helpful

MEDS = politics people perform

Sample size

141

IV to Mediators (a paths)

$\begin{array}{lrrrr} & \text { Coeff } & \text { se } & t & p \\ \text { politics } & .2106 & .0473 & 4.4571 & .0000 \\ \text { people } & .2281 & .0477 & 4.7860 & .0000 \\ \text { perform } & .1710 & .0606 & 2.8245 & .0054\end{array}$

Direct Effects of Mediators on DV (b paths)

$\begin{array}{lrrrr} & \text { Coeff } & \text { se } & t & p \\ \text { politics } & .1052 & .0983 & 1.0705 & .2863 \\ \text { people } & .3489 & .0954 & 3.6577 & .0004 \\ \text { perform } & .0332 & .0783 & .4245 & .6719\end{array}$

Total Effect of IV on DV (c path)

$\begin{array}{rrrrr} & \text { Coeff } & \text { se } & t & p \\ \text { helpful } & .2131 & .0532 & 4.0061 & .0001\end{array}$

Direct Effect of IV on DV (c' path)

$\begin{array}{rrrrr} & \text { Coeff } & \text { se } & t & p \\ \text { helpful } & .1057 & .0559 & 1.8922 & .0606\end{array}$

Fit Statistics for DV Model
$\mathrm{R}-\mathrm{sq}$
Adj R-sq
.2230
.2001
$\mathrm{F}$
9.7553
$\mathrm{df} 1$
df2
136.0000
.0000

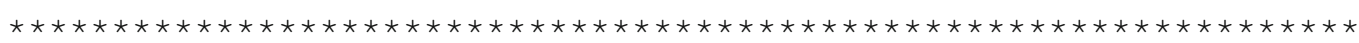

NORMAL THEORY TESTS FOR INDIRECT EFEECTS

Indirect Effects of IV on DV through Proposed Mediators (ab paths)

$\begin{array}{lrrrr} & \text { Effect } & \text { se } & \text { Z } & \text { p } \\ \text { TOTAL } & .1074 & .0312 & 3.4452 & .0006 \\ \text { politics } & .0222 & .0210 & 1.0555 & .2912 \\ \text { people } & .0796 & .0271 & 2.9366 & .0033 \\ \text { perform } & .0057 & .0133 & .4258 & .6702 \\ \text { C1 } & -.0574 & .0360 & -1.5932 & .1111 \\ \text { C2 } & .0165 & .0284 & .5801 & .5619 \\ \text { C3 } & .0739 & .0325 & 2.2730 & .0230\end{array}$




\section{APPENDIX B (Continued)}

\section{BOOTSTRAP RESULTS FOR INDIRECT EFFECTS}

$\begin{array}{lrrrr}\text { Indirect Effects of } & \text { IV } \text { on DV } & \text { through } & \text { Proposed Med } \\ & \text { Data } & \text { Boot } & \text { Bias } & \text { SE } \\ \text { TOTAL } & .1074 & .1079 & .0005 & .0268 \\ \text { politics } & .0222 & .0224 & .0003 & .0217 \\ \text { people } & .0796 & .0799 & .0003 & .0277 \\ \text { perform } & .0057 & .0056 & -.0001 & .0136 \\ \text { C1 } & -.0574 & -.0575 & -.0001 & .0407 \\ \text { C2 } & .0165 & .0168 & .0004 & .0257 \\ \text { C3 } & .0739 & .0743 & .0004 & .0350\end{array}$

Bias Corrected and Accelerated Confidence Intervals

$\begin{array}{lrr} & \text { Lower } & \text { Upper } \\ \text { TOTAL } & .0612 & .1680 \\ \text { politics } & -.0164 & .0691 \\ \text { people } & .0347 & .1481 \\ \text { perform } & -.0187 & .0361 \\ \text { C1 } & -.1508 & .0105 \\ \text { C2 } & -.0328 & .0687 \\ \text { C3 } & .0167 & .1576\end{array}$

Bias Corrected Confidence Intervals

$\begin{array}{lrr} & \text { Lower } & \text { Upper } \\ \text { TOTAL } & .0612 & .1682 \\ \text { politics } & -.0151 & .0707 \\ \text { people } & .0332 & .1430 \\ \text { perform } & -.0175 & .0383 \\ \text { C1 } & -.1420 & .0156 \\ \text { C2 } & -.0331 & .0684 \\ \text { C3 } & .0130 & .1523\end{array}$

Percentile Confidence Intervals

$\begin{array}{lrr} & \text { Lower } & \text { Upper } \\ \text { TOTAL } & .0589 & .1650 \\ \text { politics } & -.0173 & .0677 \\ \text { people } & .0312 & .1394 \\ \text { perform } & -.0201 & .0348 \\ \text { C1 } & -.1402 & .0177 \\ \text { C2 } & -.0328 & .0684 \\ \text { C3 } & .0104 & .1498\end{array}$

Level of Confidence for Confidence Intervals: 95

Number of Bootstrap Resamples: 5000

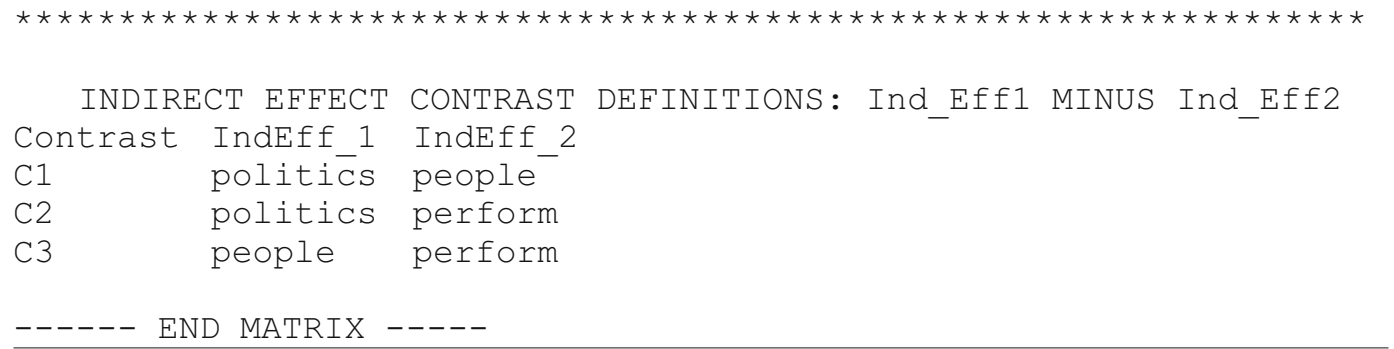

